\title{
Treatment adherence therapy in people with psychotic disorders: randomised controlled trial ${ }^{\dagger}$
}

\author{
A. B. P. Staring, M. Van der Gaag, G. T. Koopmans, J. P. Selten, J. M. Van Beveren, \\ M. W. Hengeveld, A. J. M. Loonen and C. L. Mulder
}

\section{Background}

Interventions to improve adherence to treatment in people with psychotic disorders have produced inconclusive results. We developed a new treatment, treatment adherence therapy (TAT), whose intervention modules are tailored to the reasons for an individual's non-adherence.
Aims
To examine the effectiveness of TAT with regard to service engagement and medication adherence in out-patients with psychotic disorders who engage poorly.

\section{Method}
Randomised controlled study of TAT $v$. treatment as usual (TAU) in 109 out-patients. Most outcome measurements were performed by masked assessors. We used intention-to- treat multivariate analyses (Dutch Trial Registry: NTR1159).

\section{Results}

Treatment adherence therapy $v$. TAU significantly benefited service engagement (Cohen's $d=0.48$ ) and medication adherence (Cohen's $d=0.43$ ). Results remained significant at 6-month follow-up for medication adherence. Near-significant effects were also found regarding involuntary readmissions $(1.9 \%$ v. $11.8 \%, P=0.053)$. Symptoms and quality of life did not improve.

\section{Conclusions}

Treatment adherence therapy helps improve engagement and adherence, and may prevent involuntary admission.

\section{Declaration of interest}

None.
Non-adherence with medication is prevalent in over $50 \%$ of people with psychotic disorders. ${ }^{1-3}$ If an individual stops using antipsychotic medication the risk of relapse increases three- to fivefold. ${ }^{4-6}$ Also, the risk of suicide has been found to be 3.75 times higher in people with schizophrenia who are non-adherent than in those who are adherent. ${ }^{7}$ Despite such detrimental consequences, dealing with this problem has proven difficult. Psychoeducation, for example, is not effective in improving adherence. $^{8,9}$ On the other hand, there is some evidence that cognitive-behavioural therapy is effective if it specifically targets non-adherence, ${ }^{10}$ and a meta-analysis has shown that effective strategies include practical problem-solving, and motivational techniques that link adherence to personal goals. ${ }^{8}$ Although versions of compliance therapy and adherence therapy, both including these strategies, were shown to be effective in some studies, ${ }^{11-13}$ they did not prove their usefulness in others. ${ }^{14-16}$ Research may have failed to detect effects because of a small study sample, ${ }^{15}$ relative effectiveness of the 'comparator' intervention, ${ }^{16}$ or because short-term positive effects may have been lost where only 1-year outcomes were reported. ${ }^{14}$ Based on current knowledge, the updated guidelines of the National Institute for Health and Clinical Excellence ${ }^{17}$ recommend that adherence interventions should not be used in people with schizophrenia. However, adherence interventions may be more effective when they take the large variations in reasons for non-adherence into account. This randomised controlled trial therefore measured the effectiveness of treatment adherence therapy (TAT),${ }^{18}$ in which strategies for improving adherence are tailored to individuals' situations.

\section{Method}

\section{Design and hypotheses}

This rater-masked randomised controlled trial compared a combination of TAT with treatment as usual (TAU). Our primary hypothesis was that TAT would more successfully improve service engagement and medication adherence. Our secondary hypotheses were that TAT would be more successful not only in preventing voluntary and involuntary readmission, but also in reducing symptoms and improving quality of life. We hypothesised that these effects would have four mediators: a reduction in the experience of stigma, better therapeutic alliance, increased insight and a more integrative recovery style.

\section{Procedure}

Inclusion criteria were a DSM-IV ${ }^{19}$ diagnosis of schizophrenia or schizoaffective disorder; receiving out-patient treatment; mastery of the Dutch language; at least some problems with service engagement, as defined by an average item-score of 1.25 or higher on at least two subscales of the Service Engagement Scale (SES). ${ }^{20}$ Individuals were referred when the clinician believed them to meet the criteria. In order to classify individuals according to DSM-IV, they were interviewed using the lifetime Composite International Diagnostic Interview, version 2.1 Auto. ${ }^{21}$ We then used the SES to determine whether an individual met the fourth criterion. If the person did meet it, a research assistant asked them to participate and to give written informed consent.

At baseline $\left(T_{0}\right)$, at the end of the 6-month treatment $\left(T_{1}\right)$, and at 6-month follow-up $\left(T_{2}\right)$, all respondents and their mental health professionals participated in a structured interview. These were conducted by psychology and medicine students who were masked to the participants' treatment allocations. The students received 2-day training consisting of interview role-play and of 
scoring of the instruments. After co-rating a live interview conducted by the main researcher (A.B.P.S.), they conducted two interviews under supervision. When their ratings were sufficiently consistent with those of the experienced researcher, the students conducted interviews independently, but still under supervision every other week.

Within a week of baseline assessment, participants were randomly assigned to one of the treatment conditions. This was done according to a lottery system, executed by the main researcher. Allocation was not concealed from the participants, therapists or researchers. Only the raters were masked to the treatment allocation. Because the risk existed that participants would mention the group they had been allocated to, raters were instructed to specifically ask the participant at the beginning of the interview to not tell them. This was successful; participants did not report their allocation. The participants were paid $€ 20$ for each of the three interviews. The study design was approved by the medical ethics committee of Erasmus MC University Medical Center (Dutch Trial Registry: NTR1159).

\section{Treatment adherence therapy}

Treatment adherence therapy is an intervention based on an empirical-theoretical model described previously, ${ }^{18}$ in which patient determinants of non-adherence are clustered into three groups. The first group of determinants is characterised by an individual who denies or seals over their illness and has not integrated the illness and treatment into life. The second is characterised by a person who is not satisfied with their medication because of its side-effects or low efficacy. The third is characterised by a person who lacks the cognitive skills or daily structure to participate effectively in the treatment. According to the TAT model, every individual who is non-adherent will have at least one of these clusters present, explaining the non-adherent behaviour.

The first part of the TAT manual describes the procedure for one or two initial sessions, which assess individual determinants of non-adherence. According to the clusters of determinants they detected, and after discussion with their supervisor, the therapists then filled out a decision form and chose from the three modules available: motivational interviewing, medication optimisation and behavioural training.

\section{Motivational interviewing}

The first module was an adapted form of motivational interviewing, which is intended to explore an individual's perspective and goals, developing discrepancy between current behaviour and future goals, and to help the individual with the process of placing the illness and treatment into a coherent life narrative, while avoiding resistance and emphasising freedom of choice and the individual's own responsibility. To this we added strategies for reducing selfstigmatisation such as the provision of a continuum perspective on the illness, and discussion not only of the stress-vulnerability model, but also of famous people who have struggled with psychosis.

\section{Medication optimisation}

The second module was medication optimisation. Here, a normal dosage was prescribed of an antipsychotic medication, and was then optimally tailored (which sometimes meant that a dosage was reduced) and the participant, the TAT therapist, and a family member each monitored any side-effects and psychotic symptoms in the participant. The dosage was adjusted every 6 weeks. If necessary, this procedure was repeated for various antipsychotics until an optimal medical treatment was reached. The participant clearly stated which medication they preferred, and in which dosage. To aid this decision, they used the log the participant had kept on their symptoms and side-effects. Before the start of this module, the procedure was explained to the participant in detail. The options for personal choice were emphasised, and some safety arrangements were discussed.

\section{Behavioural training}

The third option was behavioural training, which was provided to help individuals to cope with the problems caused by forgetfulness, poor executive function, poor attention and chaotic thought, all of which can cause non-adherence owing to difficulties with integrating structural treatment procedures into daily life. Training consisted of specific instructions and problem-solving strategies such as reminders, cues, reinforcement, self-monitoring tools, family or partner support and linking medication use to highly frequent behaviours.

\section{Structure of sessions}

The structure of these three modules was hierarchical. Thus, if more than one cluster of problems was present in an individual, motivational interviewing was conducted first, followed by medication optimisation and then by behavioural training. The duration and number of sessions therefore varied according to the needs of the person. In general, it took no more than 6 months.

\section{Treatment adherence therapists}

Most of the TAT therapists were psychiatric nurses. They were not the participants' own mental health professional, and gave TAT in addition to TAU. They received a full week of training and an hour's supervision every 2 weeks thereafter. To ensure treatment fidelity, all sessions were recorded and used in supervision. Although treatment fidelity was not rated with instruments, each executed session was discussed and therapeutic instructions were always given for the upcoming session. Tapes were often used to check and maintain the relevant therapeutic skills. No significant problems were encountered and the therapists were found to have cooperated with the instructions given.

\section{Treatment as usual}

Treatment as usual generally consisted of sessions with a psychiatric nurse and a psychiatrist when indicated. The sessions varied in frequency and duration, but mostly consisted of one or two sessions per month. The contents reflected overall problems the participant might encounter such as symptoms, social participation, work, daily activities and medication issues. Some participants received psychoeducation individually or in group sessions. This was recorded.

\section{Primary outcomes: service engagement and medication adherence}

\section{Service engagement}

We used the Service Engagement Scale (SES), a 14-item rating scale in which the service engagement observed is rated by the clinician most familiar with the participant. It has four subscales: availability, collaboration, help-seeking and medication compliance. As well as having good face validity and content validity, it is user-friendly, and has been shown to have good test-retest reliability in people with psychotic disorders. ${ }^{20}$ The total scale scores were used minus the subscale of medication compliance, because this subscale was included in our compiled measure of medication adherence. 


\section{Medication adherence}

We administered a semi-structured interview with participants to assess medication adherence by the independent rater. The rater normalised non-adherence as well as possible reasons for it, stressed that the obtained information would be treated confidentially and not be passed on to the participant's clinician, and enquired about the number of missed doses in the past days and weeks. Such an interviewing style has been found to produce a more valid measurement of adherence than some of the questionnaires that are used in adherence research such as the Medication Adherence Rating Scale, the Medication Adherence Questionnaire, the Drug Attitude Inventory and the Compliance Rating Scale. ${ }^{22}$ The score ranged from 0 to 4 , with higher scores indicating more problematic adherence. The scoring method was modelled after the Health of the Nation Outcome Scales. ${ }^{23}$ In this way, we used two different measures of medication adherence: one clinician-based (the SES subscale medication compliance) and one rater-based. These two measures were standardised, summed and reversed, thereby creating a compiled measure of adherence in which null scores indicated the average adherence in our study and high scores indicated good adherence. Composite measures of medication adherence help reduce the underestimation of adherence that is associated with any individual source of information. ${ }^{24}$

In our baseline data, this compiled measure of medication adherence correlated with the positive scale of the Positive and Negative Syndrome Scale (PANSS) ${ }^{25}(r=-0.23)$ and with sideeffects of antipsychotics $(r=0.23){ }^{26}$ Although relatively small, these associations provide some support for the measure's validity.

\section{Secondary outcomes}

Admissions

We documented whether, at the time the study was conducted, participants had been readmitted to a psychiatric hospital, and, if so, whether this had been voluntary or involuntary.

\section{Symptoms}

The PANSS ${ }^{25}$ is a 30 -item rating scale that is completed by the trained raters. It has three subscales: positive, negative, and general psychopathology.

\section{Quality of life}

We used the self-report EQ-5D, which has been validated in people with schizophrenia. ${ }^{27}$ On the basis of a Dutch validation study, ${ }^{28}$ the items were linearly transformed into a score ranging from -0.33 to 1.00 . High scores reflect good quality of life.

\section{Mediators}

Insight

We used Birchwood et al's self-report 8-item Insight Scale, ${ }^{29}$ whose total scores range from 0 to 12 . The scale is reliable and valid, and is easy to use within this group of participants. To have some indication of the veracity of the participants' response on this scale in our study, we calculated the Pearson correlation between this scale and the insight item of the PANSS (A12) in our baseline data. It was $-0.514(P<0.001)$, indicating an important overlap between the two.

\section{Recovery style}

Recovery style was measured using the Recovery Style Questionnaire (RSQ), ${ }^{30}$ a 39 -item self-report measure. Total scores range from 1 to 6 , low scores reflecting integration and high scores reflecting sealing-over. Broadly defined, a participant with sealing-over prefers not to think about his psychotic experience during recovery, whereas an integrator is interested in the psychotic experience and desires to put it into some coherent perspective. The RSQ is reliable and correlates highly with McGlashan's interview-based measure. ${ }^{30}$

\section{Stigma}

We used the 12-item 'perceived devaluation and discrimination' part of the self-report Stigma Scale. ${ }^{31}$ The items cover the individual's perception of common opinions about psychiatric patients such as 'Most people believe that entering a mental hospital is a sign of personal failure'. Total scores range from 1 to 4 , with higher scores indicating greater perceived stigmatisation.

\section{Therapeutic alliance}

The 36-item Working Alliance Inventory (WAI) was used to measure the alliance factor of the therapeutic relationship as it is experienced by the participant. ${ }^{32}$

\section{Statistical analyses}

Logistic regression analysis was used, with treatment allocation as the dependent variable, and with baseline demographics, medication characteristics and instrument scores as independent variables to test whether the randomisation process was conducted successfully. Next, we performed an intention-to-treat multivariate analysis (MANCOVA), in which primary outcomes were entered as dependent variables, treatment allocation as a fixed factor, and baseline SES and adherence scores as covariates. The analysis included the effects of the TAT intervention directly after it had ended $\left(T_{1}\right)$ and after 6-months of follow-up $\left(T_{2}\right)$. Significant outcomes were expressed in effect-sizes (Cohen's $d$ ) by dividing the difference in mean scores of the two treatment allocations by their pooled standard deviation. For secondary outcomes and mediators, similar analyses were used as well as Fisher's exact tests.

On the basis of the variances, error estimates and observed effects of the first 58 participants who had completed $T_{1}$, we calculated the minimum sample size necessary to achieving a power of 0.80 for our analyses. For this, we did a univariate two-group repeated-measures analysis of variance using the Greenhouse-Geisser correction to nominal degrees of freedom. It was concluded that at least 46 participants were needed in each treatment allocation to reliably detect an effect on the primary outcome measure.

\section{Results}

\section{Participants}

We screened 391 people, 195 of whom were identified as meeting the inclusion criteria. Overall, 79 refused to participate and 116 (59\%) decided to participate. The 79 who declined participation were found to engage less with services than those who decided to participate; their respective SES total scores were $23.31 \mathrm{v}$. $20.89(P<0.05$, independent samples $t$-test $)$. A further seven individuals refused further participation after baseline assessment. Age or gender differences were not found.

Table 1 portrays the characteristics of the 109 participants who were randomised. Fig. 1 shows progression through the trial. Because attrition was so small, we have performed the analyses with only the data actually gathered, rather than imputing scores for the few individuals who died or refused further participation.

\section{Randomisation}

Results of the logistic regression analysis showed that the randomisation procedure was successful; treatment allocation was not significantly predicted by any demographic variable, 


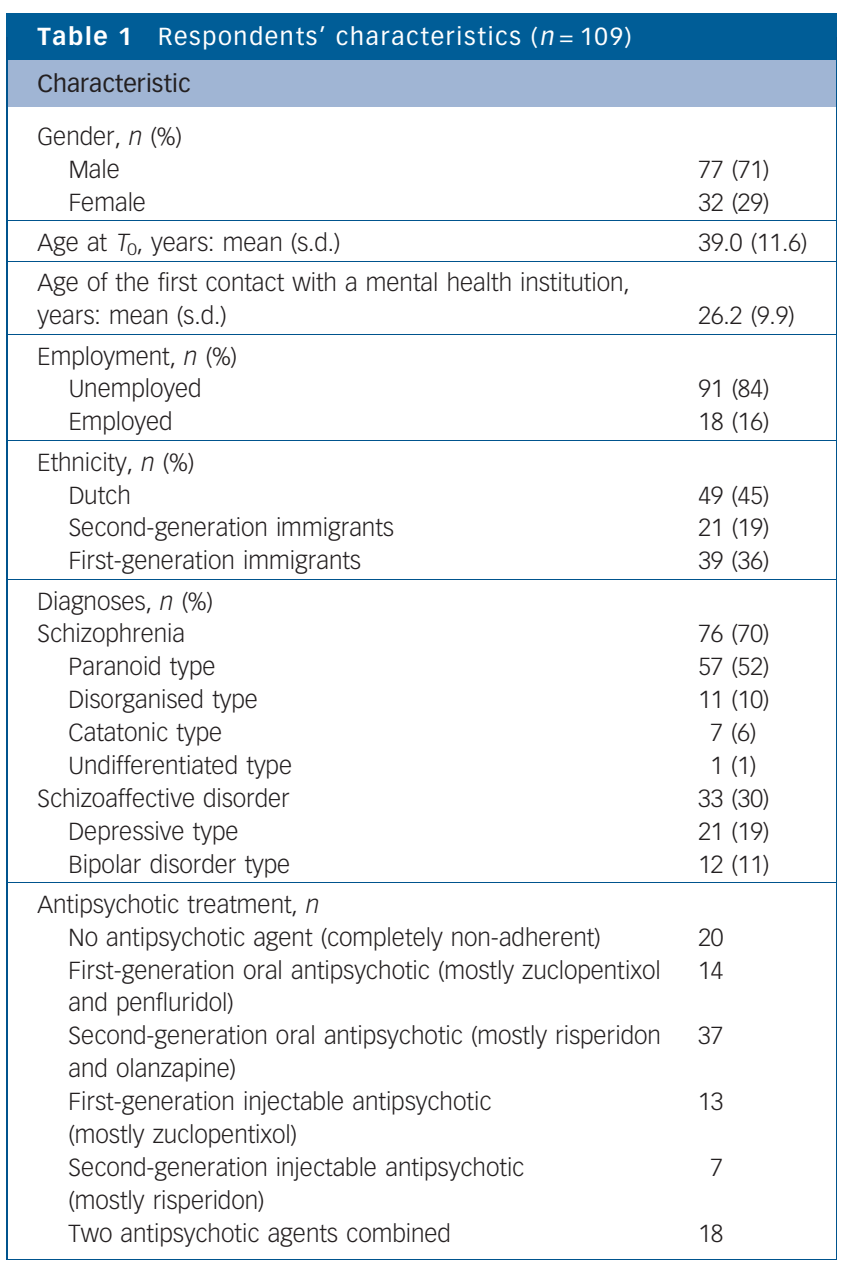

treatment characteristic or outcome variable at baseline. Importantly, the subscriptions of oral and injectable antipsychotic agents were evenly distributed between the two treatment allocations. Independent samples $t$-tests produced similar results.

\section{Therapy participation}

Of the 54 people who had been allocated to the TAT intervention, 7 dropped out after one or two sessions, and 1 left the study entirely. Another 6 did not complete the intervention; the remaining 40 participants (74\%) did. Given the basic selection of non-adherent individuals, this drop-out rate was not surprising. Those dropping out of TAT, did go on to receive TAU. Data on the primary outcome measures were gathered successfully for all 54 participants but 1 . These data were used for the intention-to-treat analyses.

In total, 40 of the 46 people who participated in the TAT intervention were given the motivational interviewing module and 6 of these 40 participants also received the medication optimisation module and 7 behavioural training. Of the remaining six, four received behavioural training, one the medication optimisation module, and one both. The mean number of TAT sessions for the 46 participants was 9.89 (s.d. $=2.72)$. This process took about 6 months.

\section{Primary outcomes: service engagement and medication adherence}

At baseline, the distributions of the outcome variables were approximately normal. As Table 2 shows, when we controlled for baseline levels in the multivariate analysis, there were

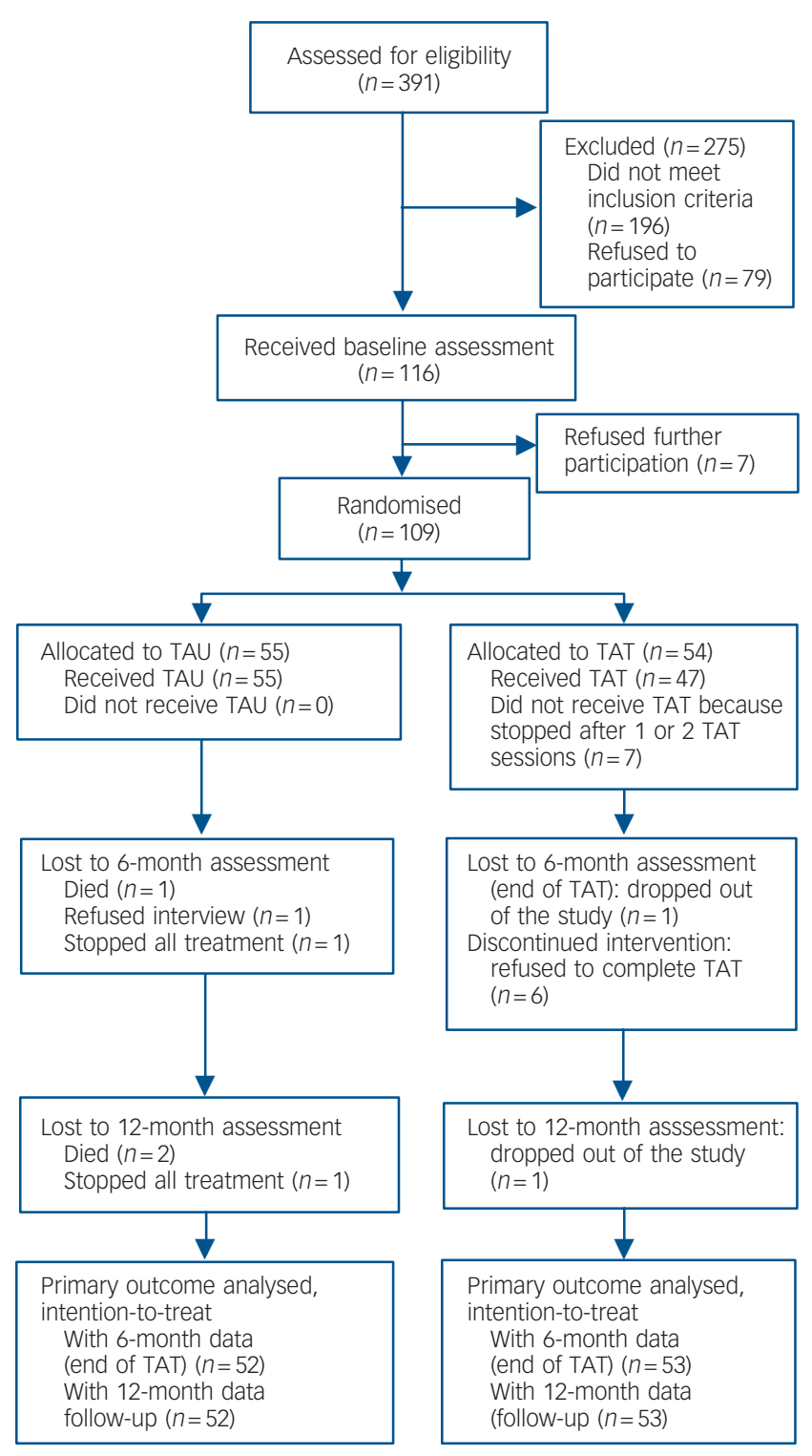

Fig. 1 Progression through the trial. TAU, treatment as usual;

TAT, treatment adherence therapy.

significant differences in service engagement and medication adherence between the two treatment conditions at the end of the TAT intervention $\left(T_{1}\right)$. Cohen's $d$ was 0.48 for service engagement and 0.41 for medication adherence. These effect sizes indicate clinical significance and can be regarded as medium effects. $^{33}$ Six months later $\left(T_{2}\right)$, Cohen's $d$ was 0.39 for service engagement and 0.30 for medication adherence, indicating that after 6 months of follow-up, the effects had reduced somewhat, and now fell within the small to medium range. Despite the smaller effect size, only the MANCOVA effects on medication adherence were still statistically significant, and not those on service engagement, which is a result of differences at baseline.

\section{Secondary outcomes}

\section{Admissions}

At baseline, all respondents were in out-patient treatment. Of those allocated to the TAT intervention group, four participants 


\begin{tabular}{|c|c|c|c|c|c|}
\hline \multirow[b]{2}{*}{ Variable } & \multirow{2}{*}{$\begin{array}{l}\text { TAT group, } \\
\text { mean (s.d.) }\end{array}$} & \multirow{2}{*}{$\begin{array}{l}\text { TAU group, } \\
\text { mean (s.d.) }\end{array}$} & \multirow[b]{2}{*}{ Cohen's $d$} & \multicolumn{2}{|c|}{ MANCOVA $^{\mathrm{b}}$} \\
\hline & & & & $\mathrm{F}$ & $P$ \\
\hline \multicolumn{6}{|c|}{ Service engagement (without the medication compliance subscale) } \\
\hline$T_{0}$ & $14.83(4.44)$ & $15.95(4.87)$ & & & \\
\hline$T_{1}$ & $10.87(6.64)$ & $14.02(6.35)$ & 0.48 & 4.995 & 0.028 \\
\hline$T_{2}$ & $10.98(6.70)$ & $13.81(7.67)$ & 0.39 & 3.561 & 0.062 \\
\hline \multicolumn{6}{|c|}{ Medication adherence } \\
\hline$T_{0}$ & $-0.09(1.55)$ & $0.02(1.92)$ & & & \\
\hline$T_{1}$ & $0.35(1.58)$ & $-0.42(1.94)$ & -0.43 & 11.43 & 0.001 \\
\hline$T_{2}$ & $0.22(1.66)$ & $-0.35(2.17)$ & -0.30 & 4.75 & 0.032 \\
\hline
\end{tabular}

(10\%) had been readmitted to a psychiatric hospital before $T_{1}$, and they did not complete the intervention. By $T_{2}$, a total of nine participants had been readmitted at least once (17\%). One of these individuals $(1.9 \%)$ had been admitted involuntarily. Of those allocated to the control group, more had been readmitted: 9 participants $(18 \%)$ before $T_{1}$; and by $T_{2}$ this increased to 14 participants $(28 \%), 6$ of whom $(11.8 \%)$ had been admitted involuntarily. Fisher's exact test showed that the difference in readmissions at any time before $T_{2}$ was not significant $(P=0.159$; one-sided $)$, and that the difference in involuntary admissions tended to significance: $1.9 \%$ in the TAT group $v$. $11.8 \%$ in the control group ( $P=0.053$; one-sided $)$.

Symptoms and quality of life

The analyses showed no effects on symptoms or quality of life (Table 3).

\begin{tabular}{|c|c|c|c|}
\hline Variable & TAT group, mean (s.d.) & TAU group, mean (s.d.) & MANCOVA $^{\mathrm{a}, \mathrm{b}}$ \\
\hline \\
\hline & \multicolumn{3}{|c|}{ PANSS positive syndrome } \\
\hline$T_{0}$ & $13.65(5.37)$ & $13.93(5.40)$ & \\
\hline$T_{1}$ & $12.92(5.20)$ & $13.10(5.75)$ & ns \\
\hline$T_{2}$ & $12.76(5.01)$ & $12.90(4.72)$ & ns \\
\hline \multicolumn{4}{|c|}{ PANSS negative syndrome } \\
\hline$T_{0}$ & $14.17(6.17)$ & $13.73(5.22)$ & \\
\hline$T_{1}$ & $12.67(5.37)$ & $13.59(5.40)$ & ns \\
\hline$T_{2}$ & $14.16(6.61)$ & $14.37(5.57)$ & ns \\
\hline \multicolumn{4}{|c|}{ PANSS general psychopathology } \\
\hline$T_{0}$ & $31.04(9.75)$ & $30.02(9.43)$ & \\
\hline$T_{1}$ & $29.63(9.04)$ & $27.27(8.09)$ & ns \\
\hline$T_{2}$ & $30.22(8.20)$ & $28.44(8.20)$ & ns \\
\hline \multicolumn{4}{|l|}{ EQ-5D } \\
\hline$T_{0}$ & $0.68(0.27)$ & $0.73(0.25)$ & \\
\hline$T_{1}$ & $0.70(0.25)$ & $0.74(0.25)$ & ns \\
\hline$T_{2}$ & $0.69(0.24)$ & $0.70(0.26)$ & ns \\
\hline \multicolumn{4}{|c|}{ Mediators } \\
\hline$T_{0}$ & $8.09(3.27)$ & $7.00(3.68)$ & \\
\hline$T_{1}$ & 7.79 (3.71) & 7.07 (3.71) & ns \\
\hline$T_{2}$ & $7.47(3.57)$ & $7.41(4.12)$ & ns \\
\hline \multicolumn{4}{|c|}{ Stigma Scale } \\
\hline$T_{0}$ & $2.74(0.51)$ & $2.77(0.45)$ & \\
\hline$T_{1}$ & $2.70(0.35)$ & $2.73(0.53)$ & ns \\
\hline$T_{2}$ & $2.78(1.21)$ & $2.43(1.24)$ & ns \\
\hline \multicolumn{4}{|c|}{ Recovery Style Questionnaire } \\
\hline$T_{0}$ & $2.57(1.11)$ & $2.79(1.26)$ & \\
\hline$T_{1}$ & $2.75(1.23)$ & $2.74(1.18)$ & ns \\
\hline$T_{2}$ & $2.78(1.21)$ & $2.43(1.24)$ & ns \\
\hline \multicolumn{4}{|c|}{ Working Alliance Inventory } \\
\hline$T_{0}$ & $141.8(23.2)$ & $139.5(22.3)$ & \\
\hline$T_{1}$ & $145.6(19.3)$ & $141.5(19.4)$ & ns \\
\hline$T_{2}$ & $143.8(24.1)$ & 140.7 (18.7) & ns \\
\hline
\end{tabular}




\section{Mediators}

The analyses showed no effects of TAT on insight, stigma, recovery style or therapeutic alliance (Table 3).

\section{Discussion}

\section{Treatment adherence therapy: observed effects and participation}

The present study compared the outcomes of two treatment conditions - TAT $v$. TAU - and found that TAT enhanced service engagement and medication adherence more successfully than TAU did. The effects were smaller at 6-month follow-up, yet still statistically significant for medication adherence.

There was a trend that the participants within the TAT condition were less often involuntarily admitted to a hospital $(1.9 \% v .11 .8 \%)-$ a difference that was almost significant. This can mean that participants in the TAT group were more likely to cooperate with their admission and in-patient treatment, whereas those in the TAU group tended to be more unwilling to comply with the recommended treatment, and to be more likely to meet the dangerousness criteria that qualified them for an involuntary admission. This is undesirable, as compulsory admission is often an if-all-else-fails measure. Even though it may have positive effects, some participants also report negative ones, such as those on therapeutic alliance, family relationships and employment prospects. ${ }^{34}$

The majority of participants found TAT acceptable. In our view, the drop-out rates from therapy were low $(26 \%)$, especially when we take account of the individuals targeted, who are often very difficult to engage in psychological treatment. On the basis of the frequent use of motivational interviewing, we also conclude that the most common reason therapists judged an individual to be engaging poorly with services was the result not of cognitive impairments but of factors such as stigma, denial and low insight.

\section{Symptoms and quality of life}

The absence of an effect on symptoms was unexpected, although other intervention studies have reported similar results. ${ }^{35}$ It may be that our sample size was too small to detect an effect on symptoms. Also, it may be that the duration and/or intensity of TAT was not enough to cause changes in symptoms and quality of life, and that we need longer or more intensive treatments. Alternatively, it may be that participants in our sample were in fact poorly adherent at baseline as a consequence of poor response to antipsychotic medication. Better adherence would then not result in large symptomatic improvements. Although adherence improved, this also did not lead to better subjective quality of life. Apparently, improving adherence per se did not lead to fewer symptoms or increases in subjective well-being in our study.

\section{Mediators}

Despite the effects of TAT on service engagement and medication adherence, it is not clear what the main mechanisms of change were. Surprisingly, insight into illness, therapeutic alliance, recovery style and the experience of stigma were all unrelated to the effects of TAT. As the baseline means of these variables allowed for improvement, given the scale ranges, a ceiling-effect was ruled out. One possibility is that our sample size was too small to detect significant effects, for example on insight, which did correlate with adherence at baseline. Another possibility is that although participants' views of treatment were changed and although participants were trained effectively in adherent behaviour, these factors were not covered by our mediator measurements.
In our study design, TAT was an add-on intervention administered by a therapist other than the participant's own clinician. This has some drawbacks and benefits. A drawback may be that TAT did not result in a better therapeutic alliance with the participants' own clinician. As we consider the approach to be patient-oriented and respectful of individuals' views, TAT may be more effective when it is administered by the individual's own clinician: it might strengthen the alliance in the regular therapeutic relationship, a factor known to positively influence outcome. ${ }^{36}$ On the other hand, a benefit of this choice in design may be that the therapist providing TAT was not confronted with other demanding topics that the person would need help with, nor was the therapist impeded by requests to change or reduce medication, which may have interfered with the adherence work.

Because good insight has been associated with depressive symptoms, low self-esteem and lower quality of life, ${ }^{37}$ improved adherence, if obtained by enhanced insight, may carry the risk of deteriorating quality of life and increasing depression. ${ }^{38}$ It is therefore noteworthy that, although we could not detect any effect of TAT on quality of life, neither did we observe a deterioration. Although TAT improved service engagement and adherence, it seems somehow to have 'bypassed' insight. Indeed, the primary focus of TAT is not on psychoeducation: instead, because it stimulates the person to develop an individual narrative into which treatment can somehow be integrated, individual motives for engagement or adherence may sometimes turn out to be different from what clinicians would find appropriate. For example, one person realised that adhering to treatment could help to avoid arguments with his partner, and this motivated him. However, he did not recognise that his symptoms and agitation increased whenever he stopped his medication, which is what caused the arguments in the first place.

\section{Limitations and strengths}

Seven limitations should be considered. First, the sample size was not large, which may have limited the power to detect treatment effects on secondary outcomes and mediators. For example, assuming that the observed difference in hospitalisation rates were non-random and would hold in a larger study, about 120 participants would be needed in each group for a difference of $18 \%$ v. $28 \%$ to become statistically significant. Second, our inclusion of many outcome variables may have increased the chances of finding a significant result. Third, the people who refused to participate in the study were engaging less with services than those included. An implication is that interventions such as TAT may not be acceptable for people with very low treatment adherence. These may benefit more from assertive treatments and direct incentives to motivate them. ${ }^{39}$

Fourth, our study design distributed attention unevenly between the two treatment allocations, which may have produced a bias. Treatment as usual mostly consisted of one or two sessions per month. Participants receiving TAT were given an average of 9.9 sessions on top of this during the course of 6 months. Thus, individuals receiving TAT were given about twice the amount of sessions of those receiving TAU. Although one study on enhancing adherence did not produce results that were more significant than those produced in a control group that received less attention, ${ }^{15}$ another found results indicating that participants improved in both the intervention and control group. ${ }^{16}$ It is therefore still unclear whether attention by itself can increase adherence, and we cannot rule out that our results were in part because of an attention bias rather than the contents of TAT.

The fifth limitation is that our measure of adherence consisted of the SES plus a one-item rating scale. This latter measure has not 
been validated in other studies, and may not be the best available. However, measuring adherence is difficult, and a gold standard is certainly lacking. ${ }^{24}$ As well as self-report and interview measures, methods of measuring adherence include pill counts, electronic methods, prescription monitoring, and saliva, plasma and urine assay tests. ${ }^{40}$ Each has its advantages and disadvantages. Pill counts, for example, are not only time consuming, but also have great potential for inaccuracy; and not only are saliva, plasma, and urine tests not possible for all drugs, they are expensive and invasive, and may also overestimate adherence for drugs that have a long half-life. ${ }^{4,8}$ However, future studies may use such alternative methods to verify our results. Hopefully, our study overcame some of the difficulties, not only because participants were asked about their adherence by interviewers who were not involved in the treatment, but also because it was made clear that the answers would be used solely for the purpose of this study and would not be communicated with caregivers. We are therefore reasonably confident that participants felt free to give honest answers about their medication adherence.

Sixth, we did not look at whether conducting the trial had an effect on what happened within TAU. The participants' treatment allocations were known to their clinicians. As a result, clinicians may have been more aware of adherence issues and have given the topic more attention, perhaps especially with participants in the control group. This may have influenced our results by, for example, increasing the effectiveness of TAU. The seventh limitation is that, although assessors were masked to the participant's treatment allocation, their clinicians were not. Therefore, the information clinicians gave on the SES was not masked from the treatment allocation, and possible bias in this measure cannot be ruled out.

Despite these limitations, the study also had at least four strengths: it had a well-defined treatment protocol that was based on an empirical-theoretical model in which strategies were tailored to the individuals' causes of non-adherence; it involved an intervention that was applied in routine settings by therapists who worked within the institutions; it used masked, independent assessors of outcome; and it had relatively high inclusion and follow-up rates of people with poor service engagement.

\section{Implications}

These are important outcomes, particularly for individuals who are non-adherent to treatment and therefore risk future relapse and readmission. The positive findings of TAT are promising and suggest optimism for further exploration. An important contributor to the effects may be that, after a person's individual situation has been assessed, TAT provides various intervention modules.

\footnotetext{
A. B. P. Staring, PhD, Erasmus University Medical Center, Department of Psychiatry, Rotterdam, and Bavo-Europoort Psychiatric Institute, Department of Outpatient Treatment, Rotterdam; M. Van der Gaag, PhD, Parnassia Psychiatric Institute, Treatment, Rotterdam; M. Van der Gaag, PhD, Parnassia Psychiatric Institute,
Department of Psychotic Disorders, The Hague, and VU University and EMGO Department of Psychotic Disorders, The Hague, and VU University and EMGO
Institute, Department of Clinical Psychology, Amsterdam; G. T. Koopmans, PhD Erasmus University, Department of Policy and Management in Health Care, Rotterdam; J. P. Selten, MD, PhD, Foundation Rivierduinen, Department of Psychotic Disorders, Leiden; J. M. Van Beveren, MD, PhD, M. W. Hengeveld, MD, PhD,

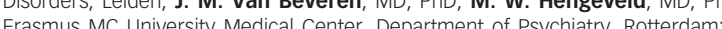
A. J. M. Loonen, MD, PhD, University of Groningen, Department of Pharmacy, Groningen; C. L. Mulder, MD, PhD, Erasmus MC University Medical Center, Department of Psychiatry, Rotterdam, and Bavo-Europoort Psychiatric Institute, Rotterdam, The Netherlands

Correspondence: Anton B. P. Staring, Research Center O3, Erasmus MC University Medical Center, Department of Psychiatry, Trouwlaan 104, 5021 WN Tilburg, The Netherlands. Email: tonnie@backwash.org

First received 4 Jan 2010, final revision 25 Apr 2010, accepted 25 May 2010
}

\section{Funding}

Funding for this study was provided by the Dutch Ministry of Health, Welfare, and Sports (ZonMw, research grant number 100-002-017).

\section{Acknowledgements}

We thank all the participating patients, caregivers and administrative, medical and paramedical staff at Erasmus MC University Medical Center, Parnassia Bavo Group, Delta Psychiatric Center, 'De Grote Rivieren' Community Psychiatric Health Center and 'Riagg Rijnmond' Community Psychiatric Health Center.

\section{References}

1 Keath SJ, Kane JM. Partial compliance and patient consequences in schizophrenia: our patients can do better. J Clin Psychiatry 2003; 64: 1308-15.

2 Thomas P. The stable patient with schizophrenia-from antipsychotic effectiveness to adherence. Eur Neuropsychopharmacol 2007; 17 (suppl 2): s115-22.

3 Weiden P, Aquila R, Standard J. Atypical antipsychotic drugs and long-term outcome in schizophrenia. J Clin Psychiatry 1996; 57 (suppl 11): s53-60.

4 Fenton WS, Blyer C, Heinssen RK. Determinants of medication compliance in schizophrenia: empirical and clinical findings. Schizophr Bull 1997; 23: 637-51.

5 Ucok A, Polat A, Cakir S, Genc A. One year outcome in first episode schizophrenia; predictors of relapse. Eur Arch Psychiatry Clin Neurosci 2006; 256: $37-43$.

6 Weiden PJ, Kozma C, Grogg A, Locklear J. Partial compliance and risk of rehospitalization among California medicaid patients with schizophrenia. Psychiatr Serv 2004; 55: 886-91.

7 Hawton K, Sutton L, Haw C, Sinclair J, Deeks JJ. Schizophrenia and suicide: systematic review of risk factors. Br J Psychiatry 2005; 187: 9-20.

8 Zygmunt A, Olfson M, Boyer CA, Mechanic D. Interventions to improve medication adherence in schizophrenia. Am J Psychiatry 2002; 159: 1653-64.

9 Lincoln TM, Wilhelm K, Nestoriuc Y. Effectiveness of psychoeducation for relapse, symptoms, knowledge, adherence and functioning in psychotic disorders: a meta-analysis. Schizophr Res 2007; 96: 2232-45.

10 Lecompte D, Pelc I. A cognitive-behavioral program to improve compliance with medication in patients with schizophrenia. Int J Ment Health 1996; 25 : 51-6,

11 Kemp R, Kirov G, Everitt B, Hayward P, David A. Randomised controlled trial of compliance therapy. 18-month follow-up. Br J Psychiatry 1998; 172: 413-9.

12 Maneesakorn S, Robson D, Gournay K, Gray R. An RCT of adherenve therapy for people with schizophrenia in Chiang Mai, Thailand. J Clin Nurs 2007; 16: 1302-12.

13 Gray R, Wykes T, Edmonds M, Leese M, Gournay K. Effect of a medication management training package for nurses on clinical outcomes for patients with schizophrenia. Cluster randomised controlled trial. Br J Psychiatry 2004; 185: 157-62.

14 O'Donnel C, Donohoe G, Sharkey L, Owens N, Migone M, Harries R, et al. Compliance therapy: a randomized controlled trial in schizophrenia. BMJ 2003; 327: 834-6.

15 Byerly MJ, Fisher R, Carmody T, Rush AJ. A trial of compliance therapy in outpatients with schizophrenia or schizoaffective disorder. J Clin Psychiatry 2005; 66: 997-1001.

16 Gray R, Leese M, Bindman J, Becker T, Burti L, David A, et al. Adherence therapy for people with schizophrenia. European multicenter randomised controlled trial. Br J Psychiatry 2006; 189: 508-14.

17 National Institute for Health and Clinical Excellence (NICE). Schizophrenia; Core Interventions in the Treatment and Management of Schizophrenia in Primary and Secondary Care (Update). NICE, 2009 (http://www.nice.org.uk/ nicemedia/pdf/CG82FullGuideline.pdf).

18 Staring ABP, Mulder CL, Van der Gaag M, Selten JP, Loonen AJM, Hengeveld MW. Understanding and improving treatment adherence in patients with psychotic disorders; a review and a proposed intervention. Curr Psychiatry Rev 2006; 2: 487-94.

19 American Psychiatric Association. Diagnostic and Statistical Manual of Mental Disorders (4th edn) (DSM-IV). APA, 1994.

20 Tait L, Birchwood M, Trower P. A new scale (SES) to measure engagement with community mental health services. J Ment Health 2002; 11: 191-8. 
21 World Health Organization. Composite International Diagnostic Interview (version 2.1 Auto). WHO, 1997.

22 Kikkert MJ, Dekker JJM, Koeter MWJ, Schene AH. The Inventory of Medication Intake (IMI): validation of an instrument for assessing adherence to antipsychotic medication. In Medication Adherence in Patients with Schizophrenia (ed MJ Kikkert): 113-32. Dissertation at the University of Amsterdam, 2010.

23 Wing JK, Beevor AS, Curtis RH, Park SB, Hadden S, Burns A. Health of the Nation Outcome Scales (HoNOS). Research and development. Br J Psychiatry 1998; 172: 11-8.

24 Kikkert MJ, Barbui C, Koeter MWJ, David AS, Leese M, Tansella M, et al. Assessment of medication adherence in patients with schizophrenia. The achilles heel of adherence research. J Nerv Men Dis 2008; 196: 274-81.

25 Kay SR, Fiszbein A, Opler LA. The positive and negative syndrome scale (PANSS) for schizophrenia. Schizophr Bull 1987; 13: 261-76.

26 Staring ABP, Mulder CL, Duivenvoorden HJ, De Haan L, Van der Gaag M. Fewer symptoms vs. more side-effects in schizophrenia? Opposing pathways between entipsychotic medication compliance and quality of life. Schizophr Res 2009; 113: 27-33.

27 Prieto L, Sacristán JA, Hormaechea JA, Casado A, Badía X, Gómez JC. Psychometric validation of a generic health-related quality of life measure (EQ-5D) in a sample of schizophrenic patients. Curr Med Res Opin 2003; 20 827-35.

28 Lamers LM, Stalmeier PFM, McDonnell J, Krabbe PFM, Van Busschbach JJ. Measuring the quality of life in cost-utility analyses: the Dutch EQ-5D tariff. Ned Tijdschr Geneeskd 2005; 149: 1574-8.

29 Birchwood M, Smith J, Drury V, Healy J, Macmillan F, Slade M. A self-report insight scale for psychosis: reliability, validity, and sensitivity to change. Acta Psychiatr Scand 1994; 89: 62-7.

30 Drayton M, Birchwood M, Trower P. Early attachment experience and recovery from psychosis. Br J Psychol 1998; 37: 269-84.
31 Link B, Struening EL, Neese-Todd S, Asmussen S, Phelan JC. On describing and seeking to change the experience of stigma. Psychiatr Rehabil Skills 2002; 6: 201-31.

32 Horvath $\mathrm{AO}$, Greenberg LS. Development and validation of the working alliance inventory. J Consult Psychol 1989; 36: 223-33.

33 Cohen J. Statistical Power Analysis for the Behavioral Sciences (2nd edn). Lawrence Erlbaum Associates, 1988.

34 O'Donoghue B, Lyne J, Hill M, Larkin C, Feeney L, O'Callaghan E. Involuntary admission from the patient's perspective. Soc Psychiatry Psychiatr Epidemiol 2010; 45: 631-8.

35 Valenstein M, Kavanagh J, Lee T, Reilly P, Dalack GW, Grabowski J, et al. Using a pharmacy-based intervention to improve antipsychotic adherence among patients with serious mental illness. Schizophr Bull 2009; Nov 21 (Epub ahead of print).

36 Hewitt J, Coffey M. Therapeutic working relationship with people with schizophrenia: literature review. J Adv Nurs 2005; 52: 561-70.

37 Staring $A B P$, Van der Gaag $M$, Van den Berge $M$, Duivenvoorden HJ, Mulder $\mathrm{CL}$. Stigma moderates the associations of insight with depressed mood, low self-esteem, and low quality of life in patients with schizophrenia spectrum disorders. Schizophr Res 2009; 115: 363-9.

38 Rathod S, Kingdon D, Smith P, Turkington D. Insight into schizophrenia: the effects of cognitive behavioural therapy on the components of insight and association with sociodemographics - data on a previously published randomised controlled trial. Schizophr Res 2005; 74: 211-9.

39 Claassen D, Fakhoury WK, Ford R, Priebe S. Money for medication: financial incentives to improve medication adherence in assertive outreach. Psychiatr Bull 2007; 31: 4-7.

40 Patel MX, David AS. Medication adherence: predictive factors and enhancement strategies. Psychiatry 2007; 6: 357-61.

\section{David Meagher}

De-Lira: to be displaced from one's furrow. Acute cognitive impairment complicates one in five hospitalisations, like a cognitive superbug penetrating healthcare environments. The kaleidoscopic symptom profile comprises generalised cognitive and neuropsychiatric disturbances. Contrasting hyperactive and hypoactive presentations complicate detection, but clinical variants share core cognitive disruptions - inattention and diminished comprehension that creates the clouded consciousness we call confusion. Half of cases occur in the context of underlying dementia with growing recognition of delirium as an accelerating and possibly causal factor in dementia. Historically understudied, recently established European and American associations can finally bring this Cinderella to the neuroscientific ball. 